\title{
Condiciones de trabajo y salud de los trabajadores inmigrantes en España. Revisión bibliográfica
}

\author{
Elena Ronda-Pérez. Andrés A. Agudelo-Suárez, María José López Jacob, Ana M. García y Fernando G. Benavides
}

Revista Española de Salud Pública, vol. 88. no. 6. Madrid nov./dic. 2014

http://dx.doi.org/10.4321/S1135-57272014000600004

DOI: 10.12961/aprl.2017.20.04.6

\section{RESUMEN}

Fundamentos: La relación entre inmigración, trabajo y salud constituye uno de los retos más importantes en salud laboral y más concretamente en España por el importante incremento de proporción inmigrante acontecido en el mercado laboral. El objetivo de esta investigación es conocer la relación entre las condiciones de trabajo y sus efectos en la salud de trabajadores inmigrantes en España.

Métodos: Revisión bibliográfica de artículos científicos originales en español e inglés en Medline y Medes (1998-2012). Se revisaron los textos completos de los artículos incluidos.

Resultados: Se incluyeron 20 estudios, 13 con metodología de investigación cuantitativa y 7 cualitativa. Los temas tratados abordaban problemas específicos de salud relacionados con el trabajo (principalmente lesiones por accidente de trabajo), incapacidad laboral y diferencias en condiciones de trabajo y empleo. Los hallazgos de los estudios mostraron mayor incidencia de lesiones por accidentes de trabajo, menores tasas de incapacidad laboral, mayor prevalencia de presentismo laboral, exposición a factores psicosociales y precariedad laboral en la población inmigrante.

Conclusiones: A pesar de la singularidad del proceso demográfico migratorio, los problemas de salud y determinantes identificados no difieren de los referenciados en otros países, en otros contextos y en otros momentos.

\section{COMENTARIO}

A finales del siglo XX, la producción científica sobre inmigración y salud en España era casi inexistente, a partir del año 2000 fue aumentando progresivamente, junto con el crecimiento exponencial de población extranjera residente ${ }^{1}$. Este escenario requería de un trabajo que retratase el estado de la cuestión, tanto en términos generales como en el ámbito de la salud laboral. El contexto requería de la metodología Scoping Review, especialmente diseñada para permitir que se recoja toda la literatura relevante sobre el tema, independientemente del diseño de los estudios, siendo especialmente apropiada para responder preguntas generales, de un contenido amplio, y de un tema complejo y no investigado antes ${ }^{2}$.

En la revisión bibliográfica que nos ocupa, sobre inmigración, trabajo y salud, la búsqueda incluyó artículos procedentes de fuentes de datos durante el periodo 2003-2008. Esta época concierne al fenómeno conocido como "la singularidad de España", debido a que de manera súbita el país se convirtió en recep-

Sección coordinada por:

Consol Serra (consol.serra@upf.edu)

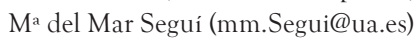

Arch Prev Riesgos Labor 2017; 20 (4): $225-226$ tor de inmigrantes, que llegaron con el objetivo de incorporarse al mercado laboral. Durante este lustro, la población extranjera residente en España casi duplicó su tamaño, pasando de menos de tres millones y medio de personas en 2003 (3.282.353) a más de 6 millones en $2008(6.092 .467)^{3}$. Las nacionalidades más representadas eran, y siguen siendo ${ }^{4}$, ecuatorianos, colombianos, marroquíes y rumanos (entonces de fuera de la UE de los 15); pero todos con el objetivo común de encontrar un trabajo y mejorar sus condiciones laborales y de vida, siendo las dificultades económicas en el país de origen la principal razón para emigrar ${ }^{5}$.

Esta revisión sobre condiciones de trabajo y salud de los trabajadores inmigrantes en España ofrece una visión general, no disponible hasta el momento, que señala principalmente dos cuestiones relevantes: Por un lado que, a pesar del aumento exponencial de trabajadores inmigrantes en España, la cantidad de publicaciones científicas sobre salud laboral en este colectivo es muy escasa. Ello explica que sólo se incluyeran veinte artículos. Por otro lado, que aunque la exposición a malas condiciones de trabajo está relacionada con mala salud para todos los trabajadores, los inmigrantes ocupados en España tienen mayor riesgo de exposición y peores resultados en salud que los autóctonos.

Los estudios más antiguos incluidos en esta revisión usaron fuentes de datos pertenecientes al periodo 2003-2005 y se centraron en accidentes laborales y factores psicosociales. Encontraron que ser inmigrante está relacionado con mayor probabilidad de 
sufrir un accidente laboral mortal y no mortal, a pesar de que está relacionado con menos bajas por enfermedad, probablemente por razones de menor protección social (trabajo informal, entre otros). Asimismo se asocia con sufrir mayores demandas en el trabajo, con tener menos control sobre los tiempos de trabajo, menos posibilidades de desarrollo profesional y con percibir menos apoyo de los compañeros y jefes. Algunos de estos estudios señalaron problemas de codificación por nacionalidad en las bases de datos abiertas disponibles en España en la evaluación de los accidentes laborales, por lo que estos resultados deben tomarse con cautela.

El resto de estudios incluidos en esta revisión utilizaron fuentes de datos pertenecientes al periodo 2006-2008, que siguen identificando a los trabajadores inmigrantes como un grupo vulnerable, especialmente en términos de exposiciones laborales de riesgo. Los estudios de los años 2006 y 2007 seguían encontrando que ser inmigrante se relaciona con accidentes y condiciones adversas en el trabajo. Los pertenecientes al año 2008 introdujeron nuevos conceptos en la investigación en salud laboral e inmigración en España, como la discriminación percibida, la situación legal, el proceso migratorio, que afecta la salud percibida de estos trabajadores.

Se considera necesaria una nueva revisión bibliográfica que ofrezca una visión general actual de los resultados en el contexto de crisis económica ya asentada, puesto que la precariedad laboral está presente en prácticamente todas las actividades económicas y golpea también a los trabajadores nacidos en España. Además, se desconocen las cifras exactas de inmigrantes retornados al país de origen y si estos cumplen un perfil determinado, que podría estar modificando el perfil de los que aún residen en España y los resultados en las investigaciones de salud y exposición, especialmente en las comparaciones con los trabajadores autóctonos. Además, el citado efecto del "trabajador sano" ha dejado de proteger a los llegados hace más de una década. Existen datos aproximados sobre el saldo migratorio, negativo desde el año $2010^{6}$, y evidencia de que las características sociodemográficas de los inmigrantes han evolucionado ${ }^{4}$. Como ejemplo, en el año 2016, más de un tercio del total de inmigrantes tenía la nacionalidad española (34,2\%), y más de la mitad de todos los inmigrantes eran de origen latinoamericano $(57,6 \%)^{3}$. A pesar de esto, en 2016 uno de cada 10 ocupados (10,9\%) tiene nacionalidad diferente a la española. Si entre 2003 y 2008 se detectó la dificultad que percibían los trabajadores inmigrantes a la hora de poder ejercer sus derechos, en 2016 desconocemos si el cambio en el estado administrativo y el tiempo de residencia podrían estar influyendo en esta percepción. Por todo esto, se hace necesaria una imagen general actualizada que describa cómo podrían estar influyendo estos cambios y en qué medida difieren de los resultados encontrados para el periodo 2003-2008, habida cuenta de la relevancia de este colectivo para la salud laboral.

\section{Ana Cayuela}

Área de Medicina Preventiva y Salud Pública. Universidad de Alicante. España.

ana.cayuela@ua.es

\section{REFERENCIAS}

1. Ronda-Pérez E, Ortiz-Barreda G, Hernando C, Vives-Cases C, Gil-González D, Casabona J. Características generales de los artículos originales incluidos en las revisiones bibliográficas sobre salud e inmigración en España. Rev Esp Salud Pública. 2014;88:675-85.

2. Arksey H, O'Malley L. Scoping studies: towards a methodological framework. International journal of social research methodology. 2005;8:19-32.

3. Instituto Nacional de Estadística. Índice interactivo INEbase, Demografía y po blación: Fenómenos demográficos. [Internet]. [citado 29 marzo 2017]. Disponible en: http://www.ine.es/dynt3/inebase/es/index.htm?padre=1894\& capsel=1895. 4. Cayuela-Mateo A, Martínez-Martínez JM, Ferrer Serret L, Felt E, Casabona I Barbarà J, Collazos Sanchez F, et al. Proyecto PELFI: Reclutamiento y características sociodemográficas de las familias inmigradas y autóctonas de las subcohortes de Alicante y Barcelona. Rev Esp Salud Pública. 2017;91:e1-e9.

5. Agudelo-Suárez AA, Ronda-Pérez E, Gil-González D, Vives-Cases C, García AM, García-Benavides F, et al. Proceso migratorio, condiciones laborales y salud en trabajadores inmigrantes en España (proyecto ITSAL). Gac Sanit. 2009;23 :115-21.

6. Instituto Nacional de Estadistica. Indice interactivo INEbas, Indicadores de crecimiento de poblacion, Saldo migratorio desde 2008. [Internet]. [citado 1 de abril de 2017]. Disponible en: http://www.ine.es/jaxiT3/Tabla.htm?t=5229.

\author{
CONDICIONES DE SEGURIDAD Y \\ SALUD LABORAL EN LA ACTIVIDAD FORESTAL \\ 30 de noviembre de 2017, Lugo (España) \\ Información: \\ Centro ISSGA de Lugo, Ronda de Fingoi, 170 \\ 27071 Lugo, España \\ Tel.: 982294300 \\ Fax.: 982294336 \\ issga.lugo@xunta.gal \\ http://issga.xunta.gal/portal/contido/o-issga/index.html
}

\section{EL CÁNCER DE ORIGEN LABORAL \\ 30 de noviembre de 2017, Pontevedra}

Información:

Centro ISSGA de Pontevedra, Camiño Coto do Coello 2.

36812 Rande-Redondela-Pontevedra, España

Tel.:886 218100

Fax.: 886218102

issga.pontevedra@xunta.gal

http://issga.xunta.gal/portal/contido/o-issga/index.html 